\title{
Densely Calculated Facial Soft Tissue Thickness for Craniofacial Reconstruction in Chinese Adults
}

Wuyang Shui ${ }^{1}$, Mingquan Zhou ${ }^{1}$, Qingqiong Deng ${ }^{1 *}$, Zhongke $\mathrm{Wu}^{1}$, Yuan $\mathrm{Ji}^{2}$, Kang $\mathrm{Li}^{3}$, Taiping $\mathrm{He}^{4}$, Haiyan Jiang ${ }^{1}$

${ }^{1}$ College of Information Science and Technology, Beijing Normal University, Beijing, 100875, China;

${ }^{2}$ Institute of Forensic Science, Ministry of Public Security, Beijing, 100038, China;

${ }^{3}$ College of Information Science and Technology, Northwest University, Xi an, 710127, China;

${ }^{4}$ Affiliated Hospital of Shaanxi University of Chinese Medicine of Shaanxi Province, Xian yang, 712000, China;

First Author: Wuyang Shui

Email: sissun@126.com

Corresponding author: Qingqiong Deng

Email: qqdeng@bnu.edu.cn

Tel: (0086)10 58804576

Address: No.1105, Area B, Kejilou building, Beijing Normal University, No. 19, XinJieKouWai St., HaiDian District, Beijing 100875, P. R. China. 


\title{
Densely Calculated Facial Soft Tissue Thickness for Craniofacial Reconstruction in Chinese Adults
}

\begin{abstract}
Craniofacial reconstruction (CFR) is used to recreate a likeness of original facial appearance for an unidentified skull; this technique has been applied in both forensics and archeology. Many CFR techniques rely on the average facial soft tissue thickness (FSTT) of anatomical landmarks, related to ethnicity, age, sex, body mass index (BMI), etc. Previous studies typically employed FSTT at sparsely distributed anatomical landmarks, where different landmark definitions may affect the contrasting results. In the present study, a total of 90198 one-to-one correspondence skull vertices are established on 171 head CT-scans and the FSTT of each corresponding vertex is calculated (hereafter referred to as densely calculated FSTT) for statistical analysis and CFR. Basic descriptive statistics (i.e. mean and standard deviation) for densely calculated FSTT are reported separately according to sex and age. Results show that $76.12 \%$ of overall vertices indicate that the FSTT is greater in males than females, with the exception of vertices around the zygoma, zygomatic arch and mid-lateral orbit. These sex-related significant differences are found at $55.12 \%$ of all vertices and the statistically age-related significant differences are depicted between the three age groups at a majority of all vertices ( $73.31 \%$ for males and $63.43 \%$ for females). Five non-overlapping categories are given and the descriptive statistics (i.e. mean, standard deviation, local standard deviation and percentage) are reported. Multiple appearances are produced using the densely calculated FSTT of various age and sex groups, and a quantitative assessment is provided to examine how relevant the choice of FSTT is to increasing the accuracy of CFR. In conclusion, this study provides a new perspective in understanding the distribution of FSTT and the construction of a new densely calculated FSTT database for craniofacial reconstruction.
\end{abstract}

Keywords: Forensic anthropology population data; facial soft tissue thickness; craniofacial reconstruction; Computed tomography (CT).

\section{Introduction}

The purpose of craniofacial reconstruction (CFR) is to recreate a likeness of facial appearance from an unidentified skull using the relationship between facial soft tissue thickness (FSTT) and the skull. In the field of forensics the CFR technique is often the final hope in recognizing and identifying a seriously decomposed cadaver [1]. In the field of archeology CFR is also available to recreate the facial appearance of historically important figures from skeletal remains [2].

Since FSTT at anatomical landmarks was first measured by puncturing cadavers with needles for CFR at the end of the 19th century, 3D manual CFR techniques have been widely used. CFR techniques can primarily be classified into three categories: the anatomical Russian method, the anthropometrical American method and the combination Manchester method [3-5]. The basis of the Russian method relies on the facial muscles, whereas the anthropometrical and combination methods require measurements of average FSTT at specific anatomical landmarks [6-9]. However, 
Stephan suggested that all of the above methods relied on both soft-tissue depth information and anatomical knowledge[10]. Multiple reconstructed appearances from the same skull may be produced based on the choice of FSTT related to the properties of the skull (such as sex, age, body mass index, ethnic, etc.) [1, 4, 11-13].

The needle puncture technique is inexpensive, easy to use and a simple form of taking FSTT measurements from cadavers [14-17]. This method has its limitations due to a degree of distortion that occurs when pressure is applied to the facial muscle, and water movement [17, 18]. The ongoing improvement of neuroimaging techniques, and non-invasive diagnostic techniques, such as ultrasound probing [12, 19-21], cephalometric radiographs[22-25], computerized tomography(CT) [26-31] and magnetic resonance imaging (MRI) [32, 33], has afforded us an opportunity to make measurements on living subjects.

Another problem with FSTT measurement is the influence of gravity while taking MRI and CT measurements, since living subjects are required to lie in the supine position. Cone beam computed tomography (CBCT) is a new reliable neuroimaging technique that can provide high-resolution cross-section images of living subjects with lower radiation and hence less danger. The advantage of CBCT is that it allows patients to remain in an upright position, therefore reducing the degree of dimension distortion [6,34-36]. Another available solution is to align two models produced by the neuroimaging and optical scanner [37, 38]. The geometric structures of both the skull and face are reconstructed from CT or MRI images, and the optical scanner is employed to acquire the facial model of the same subject in the upright position. These two facial models are then aligned and the FSTT is calculated at the specific anatomical landmarks.

Average FSTT data is the major contributor to the accuracy and reliability of CFR, which is affected by many factors, including sex [29, 31-33], age [12, 20, 28, 29], skull shape [21-23], body mass index [28, 39, 40], nutritional status [12, 17,33] and ethnic and racial group [16, 30]. Many studies have reviewed FSTT databases for various populations. Stephan et al. synthesized 62 previous published groups of FSTT data consisting of 6700 datasets [41] and updated the FSTT using T-tables [42]. Currently, FSTT datasets are available for Australian [14], Portuguese [17], Zulu [19], Egyptian [20], Caucasian [12], Czech[43], Turkish [8, 23, 33], Hungarian [38], Slovak [29], Indian [44], American [45], French [46], Japanese [21, 22], South African [30], Brazilian [15, 16], Korean [6, 34], Pakistani [47, 48] and Chinese-American [49] populations.

Several studies have reported the FSTT in the Chinese population. Dong et al. reported the FSTT at 20 anatomical landmarks on a 3D skull model taken from CT images, and statistical analysis were performed based on sex and BMI [28]. Our previous study reported the FSTT at 78 anatomical landmarks on skull models from CT images, and FSTT measurements were reported according to sex and age [31]. Chen et al. reported FSTT at 31 anatomical landmarks on MRI images, and significant differences were observed in FSTT and nasal profiles [32]. Chen et al. reported FSTT at 10 landmarks from X-ray images, and FSTT was measured in accordance with sex and age [24]. Despite these studies involving Chinese people, there was considerable variation in the average FSTT at certain anatomical landmarks, and few densely calculated FSTT data were available.

The purpose of this study is to construct a database of densely calculated FSTT for the Chinese population, taking into account sex and age. Densely calculated FSTT will provide rich detail, enabling us to investigate FSTT. The appropriate average FSTT is chosen to increase the accuracy and reliability of CFR. 
2. Materials and methods

\subsection{Materials}

A total of 171 adult individuals (100 males and 71 females) of Han ethnicity were enrolled in this study, and most of the participants were residents of Xi'an, Shaanxi province. All the samples were between the ages of 20 to 60 years and were divided into four non-overlapping age groups. Each sample has normal morphology and has never undergone any orthodontic treatment. Our research has been approved by the Institutional Review Board (IRB) of the Affiliated Hospital of the Shaanxi University of Chinese Medicine.

The medical images were acquired by means of a clinical multi-slice CT scanner system (Siemens Sensation 16) belonging to the affiliated hospital of Shaanxi university of Chinese medicine. Our previous work had extracted the exterior boundary from CT images and generated exterior models of the skull and corresponding face [4]. As the skull and corresponding face were both reconstructed from CT images and located at their local coordinate system, the Frankfurt Horizontal Plane was available as a tool to impose some standardization to remove inconsistencies due to different poses (Fig. 1) [50]. The Frankfurt Horizontal coordinate system consists of four skull landmarks: the left porion $(L p)$, the right porion $(R p)$, the left orbital $(L o)$ and the glabella $(G)$, the procedures for which were presented in our previous work [4]. A total of 78 skull landmarks have been defined and marked by the same investigator and the FSTT of each landmark was measured in our previous work [31]. In this study, we utilized these landmarks to guide 3D skull model alignment. At least three correspondence marks were required for alignment, and a fewer number of marks improved the accuracy of alignments.

\subsection{Densely calculated FSTT}

The purpose of densely calculated FSTT was to establish homologous dense one-to-one correspondence vertices on the skull for every sample, i.e. each vertex occupies the same position relative to all other vertices, and subsequently the FSTT measurements of each correspondence vertices are recorded, in other words the FSTT of correspondence vertices utilize a similar measurement orientation. The dense one-to-one correspondence vertices on the skull were established through iteratively performing the rigid and non-rigid registration of a template skull onto all the samples within the dataset. The registration allowed two skull models to be tightly matched and the closest point can be taken as the correspondence vertex. Similar to previous work [4], the generic thin plate spline (TPS) was carried out to roughly register two skull models according to anatomical landmarks, and compact support radial basis function (CSRBF) was then performed to ensure that the two skull models register tightly. As the TPS approach was sensitive to the difference in shape between the template and the target skulls, the iterative closest point (ICP) with scaling was performed to eliminate differences in position, orientation and scale before the TPS and CSRBF registration [51]. To assess the accuracy of registration, a quantitative comparison of the dimensional errors between the template and the target skulls was conducted using the open source project CloudCompare (v2.6.2). Fig. 2 shows an example the registration of the template skull (Fig. 2a) and a target skull (Fig. 2b). Two skulls are compared after being altered according to the Frankfurt Horizontal coordinate system (Fig. 2c). The geometric deviation between two skulls is visualized (Fig. 2d), where the TPS and CSRBF are used to register and the average registration error between two skulls is $1.14 \mathrm{~mm}$. The geometric deviation between two 
skulls is depicted (Fig. 2e), where ICP with scaling are conducted and the average registration error is $1.02 \mathrm{~mm}$.

After the dense skull correspondence vertices have been established, the FSTT measurements of each correspondence vertices of all the samples within the dataset were automatically calculated using our previous work [31], where the FSTT measurement was equal to the Euclidean distance between the skull vertex and the corresponding intersection point on the face. As the measurement orientation of each vertex was automatically computed, it was a challenge to ensure that the measurement orientations of correspondence vertices on every sample remained consistent. The measurement orientation defined as perpendicular to the bony surface (the normal vector on each vertex) was always adopted in the manual method, but the normal vector is sensitive to noise in the procedures of computing. To ensure consistency of the measurement orientation, let us define each skull vertex as $p_{i}=\left(x_{i}, y_{i}, z_{i}\right)$ and the measurement orientation of each vertex will be defined as $\left(\frac{x_{i}}{\sqrt{x_{i}^{2}+y_{i}^{2}}}, \frac{y_{i}}{\sqrt{x_{i}^{2}+y_{i}^{2}}}, 0\right)$, i.e. the $\mathrm{z}$ coordinate of each vertex on the skull is equal to the $\mathrm{z}$ coordinate of the corresponding intersection point on the face. The benefit of this measurement orientation was that it related to the geometric coordinate and was not insensitive to noise. Fig.3 shows an example of FSTT measurements at 78 anatomical landmarks. The yellow and red dots correspond to anatomical landmark and correspondence intersection points on the face. The line segments represent the FSTT depth and the measurement orientation of each landmark.

Only the front parts of every sample were required and the back and neck parts needed removing. A one-to-one correspondence relationship has been established between the template skull and other skull models, hence, only the front parts of the template skull were segmented. In this study, every skull consisted of a total of 90198 vertices. Fig. 4 shows the FSTT measurement of each vertex on the template skull (Fig. 4a). The corresponding intersection points on the face are calculated (Fig. 4b) and the FSTT of each vertex is visualized in the graphic format (Fig. 4c). It can be seen that the facial FSTT measurement cannot be calculated in some zones due to some voids in the skull, such as the eye-sockets, nose, and some parts of the cheek.

\subsection{Statistical analysis}

General descriptive statistics of densely calculated FSTT were recorded for different age groups of males and females, including mean and standard deviation. The average and standard deviation of densely calculated FSTT in these different groups were depicted in a graphical format on the template face using a color bar. The mean FSTT of males and females of various age groups were compared, and these deviations were visualized on the template face with a color bar.

Densely calculated FSTT were analyzed using MATLAB R2012b. In order to observe the significant differences between sexes, Student's t-test was carried out. In order to observe significant differences of FSTT between age groups, One-way analysis of variance (ANOVA) was tested. For all statistical analyses, the $p$-value of each vertex $(\mathrm{p}<0.005, \mathrm{p}<0.05$ and $\mathrm{p}>0.05)$ was recorded and any significant difference in each vertex was depicted in a graphical format on the template face with a color bar.

\subsection{Densely calculated FSTT distribution}

To understand the FSTT distribution, the FSTT measurements were classified into five categories (category 1 , category 2 , category 3 , category 4 and category 5 ) by comparing the average 
FSTT of each vertex with the given range $(0,10 \mathrm{~mm}),[10 \mathrm{~mm}, 15 \mathrm{~mm}),[15 \mathrm{~mm}, 20 \mathrm{~mm}),[20 \mathrm{~mm}$, $25 \mathrm{~mm}$ ) and FSTT value $\geq 25 \mathrm{~mm}$. Subsequently, the average FSTT of each vertex was depicted in a graphical format on the template face with a color bar, i.e. each category corresponds to some areas of the skull.

The statistic descriptives were calculated for each category across various sex and age groups. The mean and standard deviation were calculated for each vertex. The mean (SMean) and standard deviation $(S S D)$ of each category were calculated by averaging the mean and standard deviation of each vertex. We defined the local standard deviation in order to depict the degree of dispersion in the FSTT of each vertex, and how it differed from the mean FSTT of the correspondence category, instead of the mean FSTT of each vertex. The mean local standard deviations (LSD) were calculated by averaging the local standard deviation of each vertex belong to the corresponding category. The percentage of each category according to sex and age was calculated, i.e. the number of vertices in each category that accounted for the overall number of vertices.

\subsection{Estimating appearance using densely calculated average FSTT}

A reconstruction of the facial appearance of a given skull was produced based on average densely calculated FSTT, increasing the limited number of landmarks. The skull comprised a dense number of vertices, and the ends of the virtual dowels represented the facial appearance, but it lacked the eyes, nose and some parts of the cheeks. Each vertex on the reconstruction was calculated by the following equation: face $=$ skull + vector $\cdot$ tissue + organ , where we use face and skull to denote the geometric coordinates of the reconstruction and the given skull, respectively. Vector and tissue represent the measurement orientation and corresponding FSTT depth. Organ represents the eyes, nose and mouth. An appropriate average densely calculated FSTT was chosen in accordance with sex and age similar to those of the given skull (Fig. 5a). Then, the measurement orientation of each vertex was calculated and the geometric coordinates of the reconstructed facial appearance were coarsely produced (Fig. 5b). However, there were still a number of voids on the reconstructed appearance, such as the eyes, nose, and some parts of the cheek. In addition, the coarse reconstructed appearance was not smooth since the measurement orientation corresponding to the average FSTT was inconsistent with the actual measurement orientation of the given skull. To address this problem, six points were marked on the coarse reconstructed appearance and the template face (Fig. 5c). Then, the TPS approach was conducted to deform the template face to the coarse reconstructed appearance to produce a smooth appearance (Fig. 5d). From left to right, each figure showed geometric deviation in two appearances, TPS warping and the smooth appearance. Finally, suitable organs were chosen to integrate with the smooth appearance, and the holes were filled to reproduce the fine appearance (Fig. 5e). To assess the accuracy of CFR, the geometric deviation between the reconstructed and the actual appearance was calculated. The reconstructed appearance was aligned to the actual face using the ICP algorithm, and the Euclidean distances between corresponding vertices were calculated (Fig. 5f). In addition, the average reconstruction error (avgError) was defined as the average of the Euclidean distance of overall vertices.

3. Results

3.1 Average densely calculated FSTT according to sex and age

The mean and standard deviation of densely calculated FSTT were depicted on the template 
face using a color bar (Figs. 6 and 7). The mean densely calculated FSTT of each category is illustrated (Fig. 8). The results showed that the FSTT was almost distributed symmetrically along the midsagittal plane, but was not completely symmetrical. The thinner FSTT was observed at vertices located in the upper Frankfurt Horizontal plane, including the forehead, orbit, nose, etc. The thickest FSTT was observed at vertices located in the cheek region.

The comparisons of mean densely calculated FSTT in male and female groups are shown in Fig. 9. The FSTT have greater values in males across all sex and age groups, especially almost $95.36 \%$ of overall vertices have greater values at the age of 30-40, with the exception of the zygoma. Statistically significant differences between males and females are illustrated in Fig. 10. It is of note that almost $62.88 \%$ of the overall vertices can be observed to present significant differences $(p<0.05)$ at the age of 30-40. A comparison of the significant differences between the three male age groups are illustrated in Fig. 11, where almost $73.31 \%$ of overall vertices can be observed around the forehead, orbit, cheek and bottom of chin. The FSTT of females differed significantly among three age groups illustrated, where almost $63.43 \%$ of overall vertices can be observed around the forehead, orbit, cheek and left zygoma.

The general descriptive statistics of each category in various sex and age groups were reported (Tables 1-3), including mean, standard deviation, local standard deviation and the percentage for various sex and age groups. The results showed that both the largest local standard deviation and thickest FSTT corresponded to category 5, which was observed around the cheeks. The thinnest FSTT corresponded to category 1, which was observed in the upper Frankfurt Horizontal plane.

\subsection{Craniofacial reconstruction using densely calculated average FSTT}

Fig. 12 showed an example of CFR in which the appropriate average densely calculated FSTT was chosen with similar properties to the unidentified skull. The example showed a male aged 24; i.e. the average FSTT of the male and 20-30 year group was chosen. The coarse reconstructed appearance were reproduce. The geometric deviations between actual appearance and reconstructed appearance were depicted in a graphic formats. Almost $79.3 \%$ of overall vertices on the reconstructed appearance showed deviation within an error of $2.5 \mathrm{~mm}$, and the average craniofacial reconstruction error was $1.79 \mathrm{~mm}$ (Table 4). The areas whose deviation errors were greater than $2.5 \mathrm{~mm}$ can be observed around the zygoma, the zygomatic arch and the right cheek.

The average densely calculated FSTT of male and female groups were employed to create reproductions of the same given skull, and the geometric deviations were depicted in a graphic format on the coarse reconstructed appearance (Figs. 13 and 14). The average reconstruction error and percentage distribution were indicated in Tables 4 and 5 . The geometric deviation between the reconstructed appearance based on the average FSTT of various age and sex groups and the actual appearance examined the effect of FSTT variation on the reconstructed facial form, where the largest geometric deviations (above $5.0 \mathrm{~mm}$ ) were observed around parts of the zygoma, the zygomatic arch, the cheek, the base of the nose and the bottom of chin. The most accurate (within $2.5 \mathrm{~mm}$ ) were found around the forehead, nasal bridge, the lateral nose, mouth and parts of the chin. Compared to the other groups, the geometric deviation between the ages of 20-30 years in both males and females was the smallest. The geometric deviations between these two groups were similar, where $99 \%$ of overall vertices deviated within an error of $1.0 \mathrm{~mm}$ (Fig. 15).

\section{Discussion}


Forensic scientists have successfully reproduced the appearance of the unidentified skull using 3D manual and computer-aided craniofacial reconstruction, generally utilizing the average FSTT at anatomical landmarks related to age, sex, BMI, ethnicity, etc. Regardless of whether the needle puncture technique or neuroimaging measurement techniques are used, manual measurement constitutes the primary approach. Generally a limited number (less than 100) of landmarks are used, and examiners often mark landmarks and measure the FSTT at landmarks on medical images or 3D models [28, 30]. Either the FSTT of each landmark was calculated by one examiner several times [30] or various examiners measured once [28, 32]. Subsequently, measurement errors were calculated to assess the reliability using Dahlberg's formula [6] and Pearson's coefficient [28] between two measurements. Several advantages of these technologies are that the anatomical landmarks can be defined and marked by forensic experience, and the correspondence relationship of those landmarks has been established. However, the choice of anatomical landmarks does not follow standard criteria (i.e. the number and definition of anatomical landmarks may be inconsistent), and sometimes choosing different landmarks could produce contrasting findings[52]. In addition, marking anatomical landmarks is tedious work, and some anatomical landmarks are difficult in localization.

Previous studies have employed the FSTT of each vertex to illustrate the distribution of FSTT using the automatic measurements technique [31, 37]. The FSTT depth is automatically and accurately calculated once the coordinates of vertex and corresponding measurement orientation are given. However, few studies have been concerned with representing densely calculated FSTT. Inspired by establishing the dense homology one-to-one correspondence vertices on skull models and face models [53-56], this study constructs a densely calculated FSTT by establishing one-to-one correspondence vertices on skull models and automatically calculating the FSTT measurements of these correspondence vertices.

The major challenge in densely calculating the FSTT is to accurately register the template skull and other skull models, i.e. find an interpolation mapping that establishes a strict homological, one-to-one correspondence of the vertices. Anatomical landmarks are often used to guide registration. The ICP algorithm is a popular rigid registration, in which the correspondence point and transformation are iteratively calculated until the registration error is less than threshold. Actually, rigid mapping does not guarantee the accuracy of skull model registration. Non-rigid deformation is a tool that is available to allow various models to tightly match [57]. The TPS algorithm has been widely used to carry out model registration, but TPS is a global interpolation function that is easily affected by geometric differences. The ICP with scaling algorithm is employed to reduce the shape size difference, and then global TPS and local CSRBF algorithms have been carried out to deform two models until the deformation no longer changes. These steps improve the registration accuracy between the template and the target skulls to enable densely calculated FSTT as accurate as possible.

Several conclusions have been reached about the differences between male and female groups by the mean and standard deviation of FSTT at anatomical landmarks in prior studies [14, 17, 28, 29, 32, 39, 49, 58]. For example, the region located over the Frankfurt Horizontal plane is thinner than that of the lower part, and the FSTT is greater in males than in females; significant differences at some landmarks were examined between males and females, and the FSTT on the lower region had greater variation when compared to the upper region. Nevertheless, Stephan et al. found that male and female tissue thickness differed slightly [41, 59]. For the Chinese population, 
Dong et al. found that males had thicker FSTT than females at most of the landmarks, and one third of subjects showed a statistically significant difference between males and females [28]. Chen et al. concurred that males had thicker FSTT than females at most of the landmarks, and the thickest FSTT occurred in males and females at the ages of 45-59 and 35-44 [32]. The present study is from the viewpoint of densely calculated FSTT, providing a new perspective to verify the FSTT according to both sex and age. It has been found that males have thicker FSTT than females at majority of the vertices. The thinnest FSTT of males and females occur in the 20-30 age group. The most notable variation in FSTT is located around area of the cheek, since the standard deviations and defined local standard deviation values are greater in these zones.

As the literature $[6,8]$ shows, the zygoma, the zygomatic arch and mid-lateral orbit showed a smaller FSTT in males, assuming that the FSTT at the landmarks can represent the FSTT of the correspondence area. In the present study, we provide the average densely calculated FSTT for males and females and find that females have thicker FSTT than males around the zygoma area, zygomatic arch and mid-lateral orbit. It is worth mentioning that almost $95.36 \%$ of vertices of males are thicker at age of 30-40 years and $43.85 \%$ of overall vertices in females are thicker at age of 40-50 years. Almost 55.12\% of overall vertices are observed to exhibit significant variation according to sex, whereas few vertices show differences for sex in the age groups of 20-30 and 40-50. Hence, sexual dimorphism may not play a great role in influencing the results of craniofacial reconstruction. Previous studies showed that age-related changes in tissues are highly variable[7, 32, 60], but the literature [33] indicates that age differences are not directly at landmarks due to age-related differences in fat distributions. In our age-group classification, the majority of vertices show significant differences between the three age groups, which indicates age may play a dominant role in affecting the results of CFR.

In forensic science, craniofacial reconstruction is a powerful tool for recreating the appearance of the victim, when only skeletal remains exist. The average tabulated tissue thickness at anatomical landmarks is utilized in both manual and computer-aided CFR. To quantitatively assess the accuracy and reliability of CFR, geometric morphometric comparison has been widely used to compare the computer-aided reconstruction and actual face $[3,27,35,50,61]$. In these studies, the average FSTT at various landmarks were attached to the surface of the skull, and the facial muscles were imported and placed on the skull using computer software (such as 3ds Max from Autodesk). The eyes, ears and nose were fixed onto the reconstructed appearance, and the facial features were sculpted to improve the accuracy. Wilkinson et al. found that $60 \%$ of the reconstructed face deviated no more than $2.5 \mathrm{~mm}$ for males and $51.8 \%$ for females. The right forehead, upper and lower cheeks, and some of the nasal tips deviated between 2.5 and $5 \mathrm{~mm}$ [27]. Lee et al. found $90 \%$ of those for the male and $75 \%$ of those for the female deviated within 5.0 $\mathrm{mm}$ [3]. Short et al. showed a deviation error level of $2.5 \mathrm{~mm}$, and the areas with the largest errors were observed around the cheek and zygoma region [35]. Decker et al. demonstrated that the cheeks and area around the mouth have a larger deviation [50]. In the present study, average densely calculated FSTT related to sex and age is used to reproduce appearance, and the geometric deviation is calculated to examine the effect of FSTT variation on CFR. The results indicate that the smallest geometric deviations are observed around the forehead and the largest geometric deviations are observed around the cheek, zygoma and zygomatic arch.

There are a number of voids on the reconstruction as a result of holes in the cheek and where organs (mouth, eyes and nose) are missing. Hence, hole filling and integration of the missing 
organs will provide a more realistic appearance. The holes on the cheek can be filled according to the geometric shape of the neighboring area in terms of interpolation function and the choice of organs will affect the accuracy of craniofacial reconstruction. Previous studies have presented the geometric shape of the mouth, nose and eyes relative to the bony structure of the skull $[9,18,62$, 63]. The mouth is related to the occlusion of the teeth and the mouth corners positioned below the infraorbital foramina. The nasal tip was defined using the pronasale and nasal spine line. The eye shape is determined by the relative position of the orbital tubercle and the lacrimal fossa. In this study, various organs have been manually segmented from face models and a bank of organ models contains a number of mouths, eyes and noses. The appropriate organ is chosen by forensic experts and subsequently imported and placed on to the reconstructed appearance. To enable the chosen organs and reconstructed appearance to closely match, the local non-rigid registration method has been carried out to deform the geometric shape of the organs.

Multiple reconstructions from the same skull can be produced using tabulated soft tissue thickness to provide a wide range of possible appearances [1, 11-13]. De Greef et al. produced and as proposed by various studies [12]. Starbuck and Ward compared the reconstructed appearance using FSTT at landmarks in emaciated, normal and obese subjects [13]. Lee et al. produced a more accurate reconstruction than previous ones by using an updated average FSTT [61]. It is uncertain whether employing appropriate average FSTT related to sex and age will lead to increased accuracy. In the present study, we produce multiple reconstructions using average densely calculated FSTT for various groups. In our sample, $79.3 \%$ of overall vertices show geometric deviations that are less than $2.5 \mathrm{~mm}$, and $99.7 \%$ of overall vertices showed deviations that were less than $5.0 \mathrm{~mm}$ using the male 20-30 age group with similar skull properties. The worst sample was the male 30-40 age group, for which only $34.6 \%$ show deviations within 2.5 $\mathrm{mm}$, and $21.8 \%$ of overall vertices show deviations that are larger than $5.0 \mathrm{~mm}$. It is worth mentioning that the appropriate average FSTT measurements are important to increase the accuracy of CFR.

One limitation of this study concerns the FSTT acquired from CT images in supine decubitus. The FSTT are divergent from the actual values because of the force of gravity, especially in the regions of the cheek. In addition, a limited number of living subjects is employed for each sex and age group. Further research will require a larger sample size of living subjects.

\section{Conclusion}

This study provides a new set of densely calculated facial soft tissue thickness data for the Chinese adult population, taking sex and age into account. These FSTT data can be made available for the craniofacial reconstruction of Chinese adults. A quantitative assessment is conducted to evaluate geometric deviations and examine the effect of FSTT variation on the reconstructed facial form. In addition, this study provides a new perspective in understanding and examining densely calculated FSTT according to sex and age.

[1] Claes P, Vandermeulen D, De Greef S, Willems G, Clement JG, Suetens P. Computerized craniofacial reconstruction: Conceptual framework and review. Forensic science international. 2010;201:138-45.

[2] Lee WJ, Yoon AY, Mi KS, Wilkinson CM, Dong HS. The archaeological contribution of forensic craniofacial reconstruction to a portrait drawing of a Korean historical figure. Journal of 
Archaeological Science. 2014;49:228-36.

[3] Lee WJ, Wilkinson CM, Hwang HS. An accuracy assessment of forensic computerized facial reconstruction employing cone-beam computed tomography from live subjects. Journal of forensic sciences. 2012;57:318-27.

[4] Deng Q, Zhou M, Shui W, Wu Z, Ji Y, Bai R. A novel skull registration based on global and local deformations for craniofacial reconstruction. Forensic science international. 2011;208:95-102.

[5] Stephan CN, Henneberg M. Building faces from dry skulls: are they recognized above chance rates? Journal of forensic sciences. 2001;46:432-40.

[6] Hwang HS, Park MK, Lee WJ, Cho JH, Kim BK, Wilkinson CM. Facial soft tissue thickness database for craniofacial reconstruction in Korean adults. Journal of forensic sciences. 2012;57:1442-7.

[7] Chung JH, Chen HT, Hsu WY, Huang GS, Shaw KP. A CT-scan database for the facial soft tissue thickness of Taiwan adults. Forensic science international. 2015;253:132 e1-11.

[8] Bulut O, Sipahioglu S, Hekimoglu B. Facial soft tissue thickness database for craniofacial reconstruction in the Turkish adult population. Forensic science international. 2014;242:44-61.

[9] VaneZis M. Forensic facial reconstruction using 3-D computer graphics: evaluation and improvement of its reliability in identification: University of Glasgow; 2008.

[10] Stephan CN. Beyond the sphere of the English facial approximation literature: ramifications of German papers on western method concepts. Journal of forensic sciences. 2006;51:736-9.

[11] Wilkinson C. Computerized forensic facial reconstruction. Forensic science, medicine, and pathology. 2005;1:173-7.

[12] De Greef S, Claes P, Vandermeulen D, Mollemans W, Suetens P, Willems G. Large-scale in-vivo Caucasian facial soft tissue thickness database for craniofacial reconstruction. Forensic science international. 2006;159 Suppl 1:S126-46.

[13] Starbuck JM, Ward RE. The affect of tissue depth variation on craniofacial reconstructions. Forensic science international. 2007;172:130-6.

[14] Domaracki M, Stephan CN. Facial soft tissue thicknesses in Australian adult cadavers. Journal of forensic sciences. 2006;51:5-10.

[15] de Almeida NH, Michel-Crosato E, de Paiva LAS, Biazevic MGH. Facial soft tissue thickness in the Brazilian population: New reference data and anatomical landmarks. Forensic science international. 2013;231:404. e1-. e7.

[16] Tedeschi-Oliveira SV, Melani RF, de Almeida NH, de Paiva LA. Facial soft tissue thickness of Brazilian adults. Forensic science international. 2009;193:127 e1-7.

[17] Codinha S. Facial soft tissue thicknesses for the Portuguese adult population. Forensic science international. 2009;184:80 e1-7.

[18] Wilkinson C. Facial reconstruction--anatomical art or artistic anatomy? Journal of anatomy. 2010;216:235-50.

[19] Aulsebrook W, Becker P, İşcan MY. Facial soft-tissue thicknesses in the adult male Zulu. Forensic science international. 1996;79:83-102.

[20] El-Mehallawi IH, Soliman EM. Ultrasonic assessment of facial soft tissue thicknesses in adult Egyptians. Forensic science international. 2001;117:99-107.

[21] Utsuno H, Kageyama T, Uchida K, Yoshino M, Oohigashi S, Miyazawa H, et al. Pilot study of facial soft tissue thickness differences among three skeletal classes in Japanese females. Forensic science international. 2010;195:165 e1-5.

[22] Utsuno H, Kageyama T, Deguchi T, Yoshino M, Miyazawa H, Inoue K. Facial soft tissue thickness 
in Japanese female children. Forensic science international. 2005;152:101-7.

[23] Kurkcuoglu A, Pelin C, Ozener B, Zagyapan R, Sahinoglu Z, Yazici AC. Facial soft tissue thickness in individuals with different occlusion patterns in adult Turkish subjects. Homo : internationale Zeitschrift fur die vergleichende Forschung am Menschen. 2011;62:288-97.

[24] Chen TY, Jia-Shu YU, Shan Z. The measurement of craniofacial soft tissue thickness in individuals of Chinese Tujia nationality. Chinese Journal of Forensic Medicine. 2005;20:213-5.

[25] Zhou S, Ou P, Yu M, Guo X, Shao C, He L, et al. Cephalometric analysis of the soft tissue profile in Hunan Han adults with normal occlusion. Zhong nan da xue xue bao Yi xue ban = Journal of Central South University Medical sciences. 2015;40:398-405.

[26] Phillips V, Smuts N. Facial reconstruction: utilization of computerized tomography to measure facial tissue thickness in a mixed racial population. Forensic science international. 1996;83:51-9.

[27] Wilkinson C, Rynn C, Peters H, Taister M, Kau CH, Richmond S. A blind accuracy assessment of computer-modeled forensic facial reconstruction using computed tomography data from live subjects. Forensic Science Medicine \& Pathology. 2006;2:179-87.

[28] Dong Y, Huang L, Feng Z, Bai S, Wu G, Zhao Y. Influence of sex and body mass index on facial soft tissue thickness measurements of the northern Chinese adult population. Forensic science international. 2012;222:396 e1-7.

[29] Panenkova P, Benus R, Masnicova S, Obertova Z, Grunt J. Facial soft tissue thicknesses of the mid-face for Slovak population. Forensic science international. 2012;220:293 e1-6.

[30] Cavanagh D, Steyn M. Facial reconstruction: soft tissue thickness values for South African black females. Forensic science international. 2011;206:215 e1-7.

[31] Shui WY, Zhou MQ, Yuan JI. Facial Soft Tissue Thickness Measurement and Its Application in Craniofacial Reconstruction. Acta Anthropologica Sinica. 2013;32:345-53.

[32] Chen F, Chen Y, Yu Y, Qiang Y, Liu M, Fulton D, et al. Age and sex related measurement of craniofacial soft tissue thickness and nasal profile in the Chinese population. Forensic science international. 2011;212:272 e1-6.

[33] Sipahioglu S, Ulubay H, Diren HB. Midline facial soft tissue thickness database of Turkish population: MRI study. Forensic science international. 2012;219:282 e1-8.

[34] Lee WJ, Wilkinson CM, Hwang HS. An Accuracy Assessment of Forensic Computerized Facial Reconstruction Employing Cone - Beam Computed Tomography from Live Subjects. Journal of forensic sciences. 2012;57:318-27.

[35] Short LJ, Khambay B, Ayoub A, Erolin C, Rynn C, Wilkinson C. Validation of a computer modelled forensic facial reconstruction technique using CT data from live subjects: a pilot study. Forensic science international. 2014;237:147. e1-. e8.

[36] Ruiz NAP. Facial soft tissue thickness of Colombian adults. Forensic science international. 2013;229:160. e1-. e9.

[37] Prieels F, Hirsch S, Hering P. Holographic topometry for a dense visualization of soft tissue for facial reconstruction. Forensic science, medicine, and pathology. 2009;5:11-6.

[38] Kustar A, Forro L, Kalina I, Fazekas F, Honti S, Makra S, et al. FACE-R--a 3D database of 400 living individuals' full head CT- and face scans and preliminary GMM analysis for craniofacial reconstruction. Journal of forensic sciences. 2013;58:1420-8.

[39] De Greef S, Vandermeulen D, Claes P, Suetens P, Willems G. The influence of sex, age and body mass index on facial soft tissue depths. Forensic science, medicine, and pathology. 2009;5:60-5.

[40] Baillie LJ, Mirijali SA, Niven BE, Blyth P, Dias GJ. Ancestry and BMI Influences on Facial Soft 
Tissue Depths for A Cohort of Chinese and Caucasoid Women in Dunedin, New Zealand. Journal of forensic sciences. 2015;60:1146-54.

[41] Stephan CN, Simpson EK. Facial soft tissue depths in craniofacial identification (part I): An analytical review of the published adult data. Journal of forensic sciences. 2008;53:1257-72.

[42] Stephan CN. The application of the central limit theorem and the law of large numbers to facial soft tissue depths: T-Table robustness and trends since 2008. Journal of forensic sciences. 2014;59:454-62.

[43] Drgáčová A, Dupej J, Velemínská J. Facial Soft Tissue Thicknesses in the Present Czech Population. Forensic science international. 2016.

[44] Saxena T, Panat SR, Sangamesh N, Choudhary A, Aggarwal A, Yadav N. Facial soft tissue thickness in North Indian adult population. J Indian Acad Oral Med Radiol. 2012;24:121-5.

[45] Parks CL, Richard AH, Monson KL. Preliminary assessment of facial soft tissue thickness utilizing three-dimensional computed tomography models of living individuals. Forensic science international. 2014;237:146. e1-. e10.

[46] Guyomarc'h P, Santos F, Dutailly B, Coqueugniot H. Facial soft tissue depths in French adults: variability, specificity and estimation. Forensic science international. 2013;231:411. e1-. e10.

[47] Jeelani W, Fida M, Shaikh A. Facial soft tissue thickness among various vertical facial patterns in adult Pakistani subjects. Forensic science international. 2015;257:517. e1-. e6.

[48] Jeelani W, Fida M, Shaikh A. Facial soft tissue thickness among three skeletal classes in adult Pakistani subjects. Journal of forensic sciences. 2015;60:1420-5.

[49] Chan WN, Listi GA, Manhein MH. In vivo facial tissue depth study of Chinese-American adults in New York City. Journal of forensic sciences. 2011;56:350-8.

[50] Decker S, Ford J, Davy-Jow S, Faraut P, Neville W, Hilbelink D. Who is this person? A comparison study of current three-dimensional facial approximation methods. Forensic science international. 2013;229:161. e1-. e8.

[51] Ying S, Peng J, Du S, Qiao H. A scale stretch method based on ICP for 3D data registration. Automation Science and Engineering, IEEE Transactions on. 2009;6:559-65.

[52] Berar M, Tilotta FM, Glaunès JA, Rozenholc Y. Craniofacial reconstruction as a prediction problem using a Latent Root Regression model. Forensic science international. 2011;210:228-36.

[53] Claes P, Walters M, Shriver MD, Puts D, Gibson G, Clement J, et al. Sexual dimorphism in multiple aspects of 3D facial symmetry and asymmetry defined by spatially dense geometric morphometrics. Journal of anatomy. 2012;221:97-114.

[54] Claes P, Walters M, Clement J. Improved facial outcome assessment using a 3D anthropometric mask. International journal of oral and maxillofacial surgery. 2012;41:324-30.

[55] Deng Q, Zhou M, Wu Z, Shui W, Ji Y, Wang X, et al. A regional method for craniofacial reconstruction based on coordinate adjustments and a new fusion strategy. Forensic science international. 2016;259:19-31.

[56] Paysan P, Lüthi M, Albrecht T, Lerch A, Amberg B, Santini F, et al. Face Reconstruction from Skull Shapes and Physical Attributes. DAGM-Symposium: Springer; 2009. p. 232-41.

[57] Turner WD, Brown RE, Kelliher TP, Tu PH, Taister MA, Miller KW. A novel method of automated skull registration for forensic facial approximation. Forensic science international. 2005;154:149-58.

[58] Jiménez-Arenas JM, Esquivel JA. Comparing two methods of univariate discriminant analysis for sex discrimination in an Iberian population. Forensic science international. 2013;228:175. e1-. e4. 
[59] Stephan CN, Norris RM, Henneberg M. Does sexual dimorphism in facial soft tissue depths justify sex distinction in craniofacial identification. Journal of forensic sciences. 2005;50:513-8.

[60] Wilkinson C. Forensic facial reconstruction: Cambridge University Press; 2004.

[61] Lee WJ, Wilkinson CM, Hwang HS, Lee SM. Correlation Between Average Tissue Depth Data and Quantitative Accuracy of Forensic Craniofacial Reconstructions Measured by Geometric Surface Comparison Method. Journal of forensic sciences. 2015;60:572-80.

[62] Albert AM, Ricanek K, Jr., Patterson E. A review of the literature on the aging adult skull and face: implications for forensic science research and applications. Forensic science international. 2007;172:1-9.

[63] Maltais Lapointe G, Lynnerup N, Hoppa RD. Validation of the new interpretation of Gerasimov's nasal projection method for forensic facial approximation using CT data. Journal of forensic sciences. 2015 . 


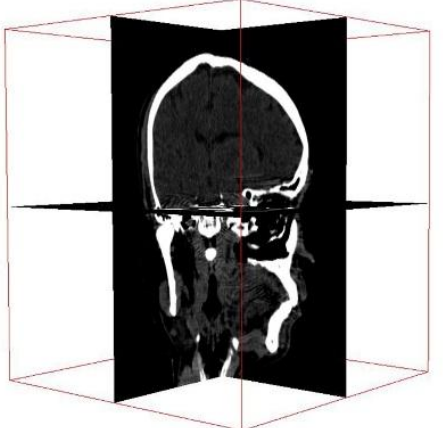

(a)

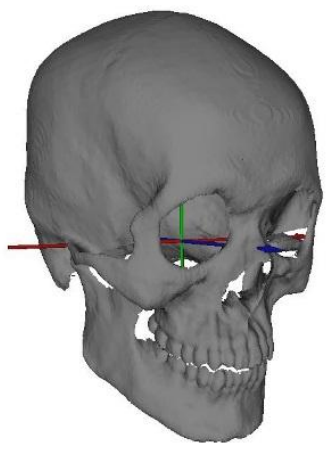

(b)

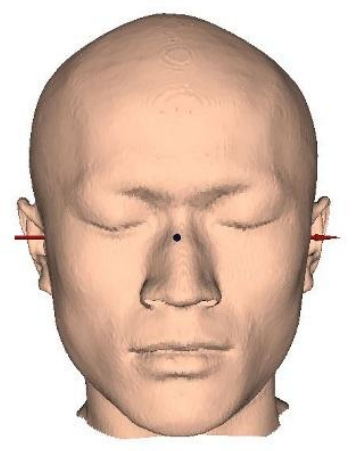

Fig. 1. The skull and corresponding face in the Frankfurt coordinate system. (a) CT images (transverse view, sagittal view and coronal view); (b) Skull and corresponding face transformed from local coordinate system to Frankfurt coordinate system.

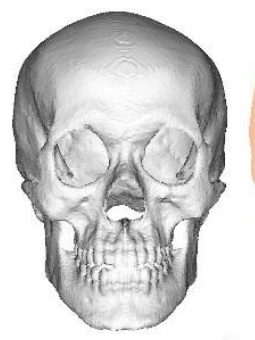

(a)
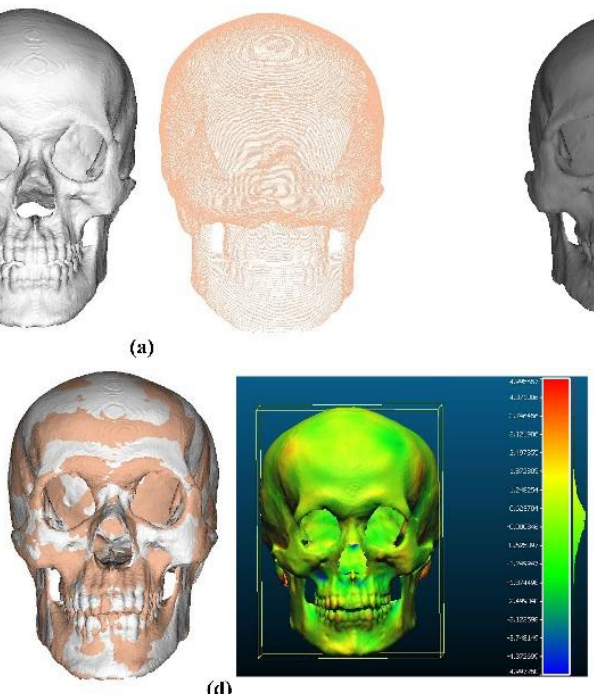

(d)

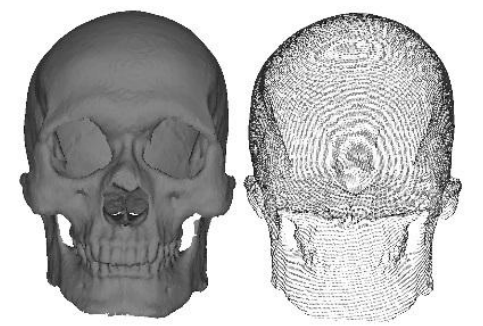

(b)

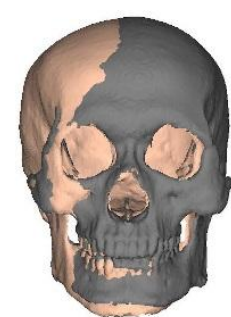

(c)

Fig. 2. Skull model registration. (a) template skull; (b) target skull; (c) two skull models located in the common coordinated system; (d) two skull model registration using RBF and CSRBF; (e) two skull model registration considering scaling before non-rigid registration performance.

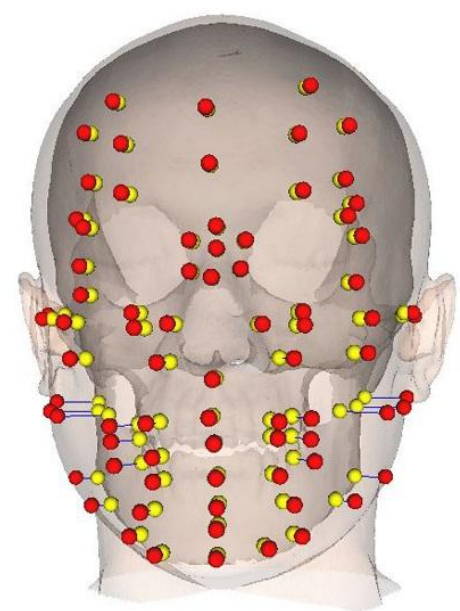

Fig. 3. FSTT measurements at 78 anatomical landmarks. 


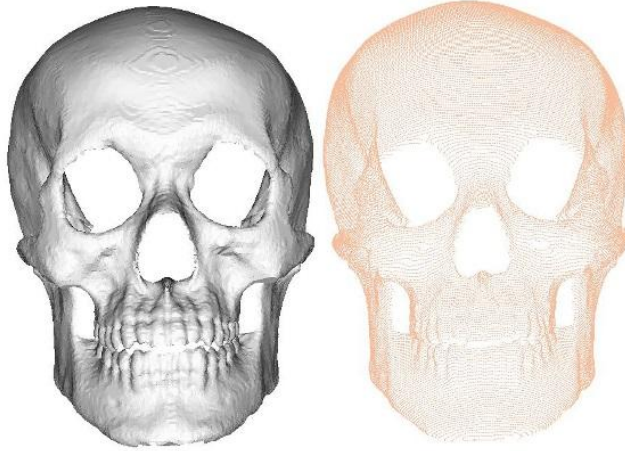

(a)

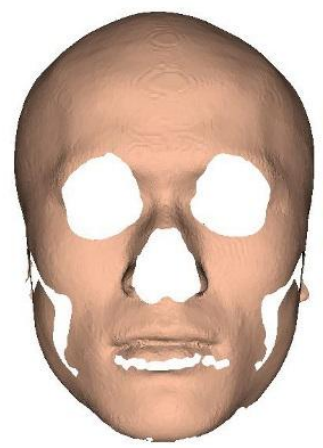

(b)

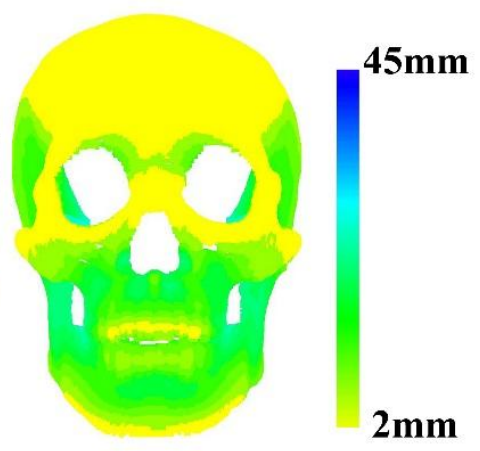

(c)

Fig. 4. Facial soft tissue thickness measurement of every vertex. (a) Template skull (triangle meshes and point clouds); (b) The corresponding intersection points on the face; (c) FSTT measurement of every vertex.

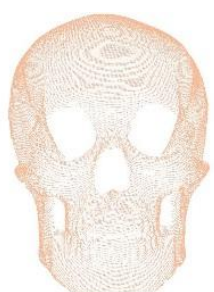

(a)

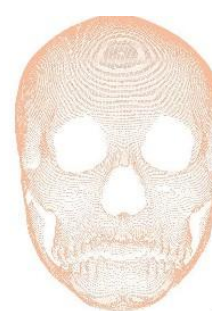

(b)
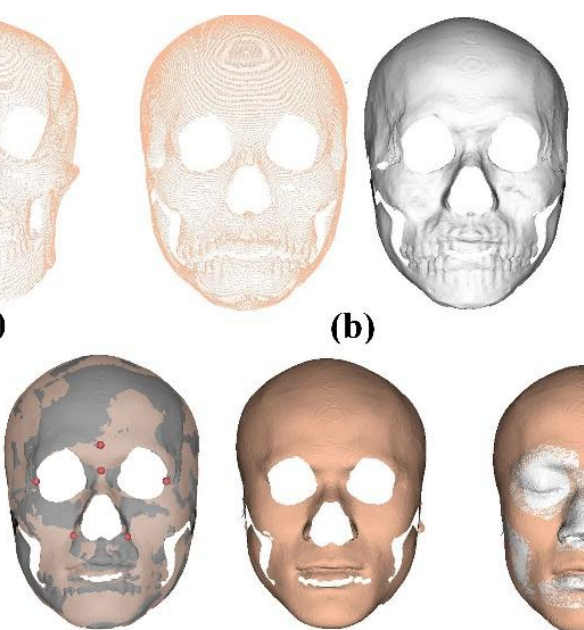

(d)

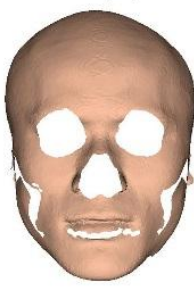

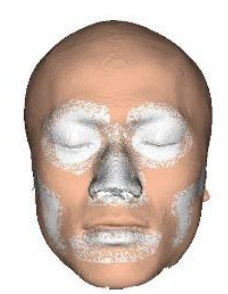

(e)

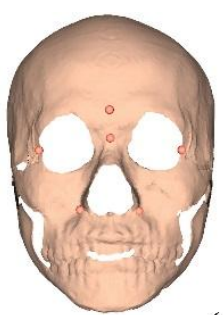

(c)

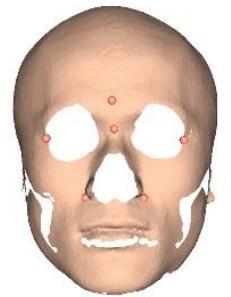

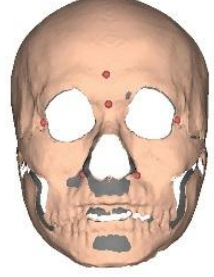

Fig. 5. Estimating the appearance. (a) The given skull; (b) Coarse reconstructed appearance using average densely calculated FSTT; (c) Six points on the reconstruction and the template face; (d) TPS warping, from left to right, geometric deviation of two models, TPS warping and smooth appearance; (e) Hole filling and organ integration; (f) Geometric deviation between the reconstructed and actual appearance.
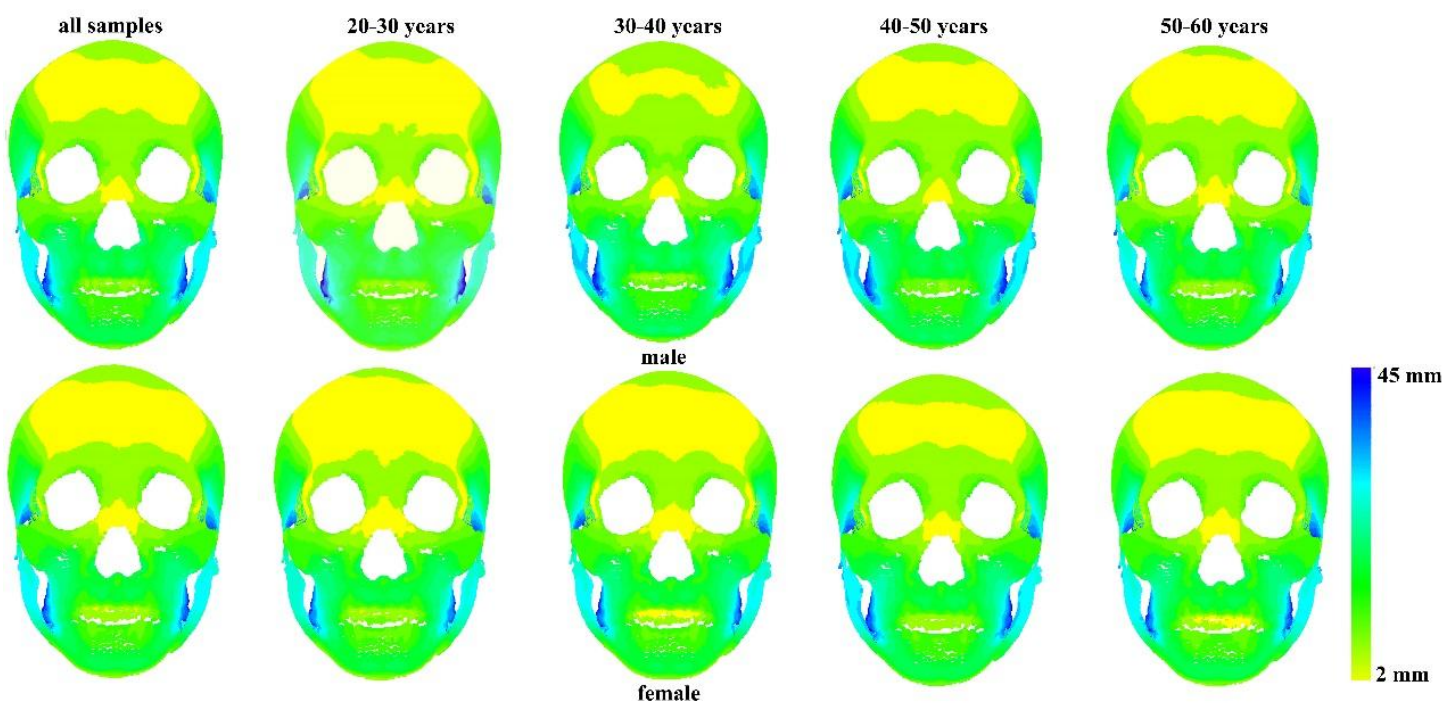
Fig. 6. Average densely calculated FSTT for various groups related to sex and age.
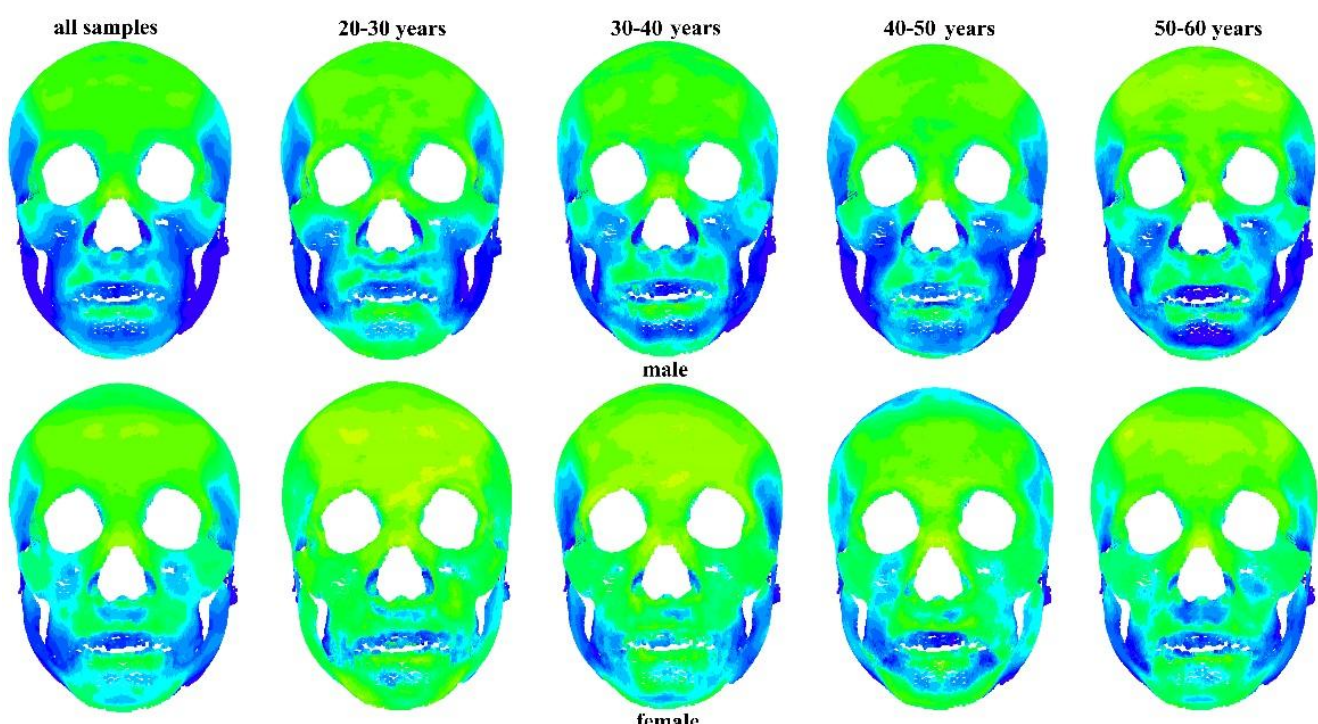

Fig. 7. Standard deviation of densely calculated FSTT for various groups according to sex and age.

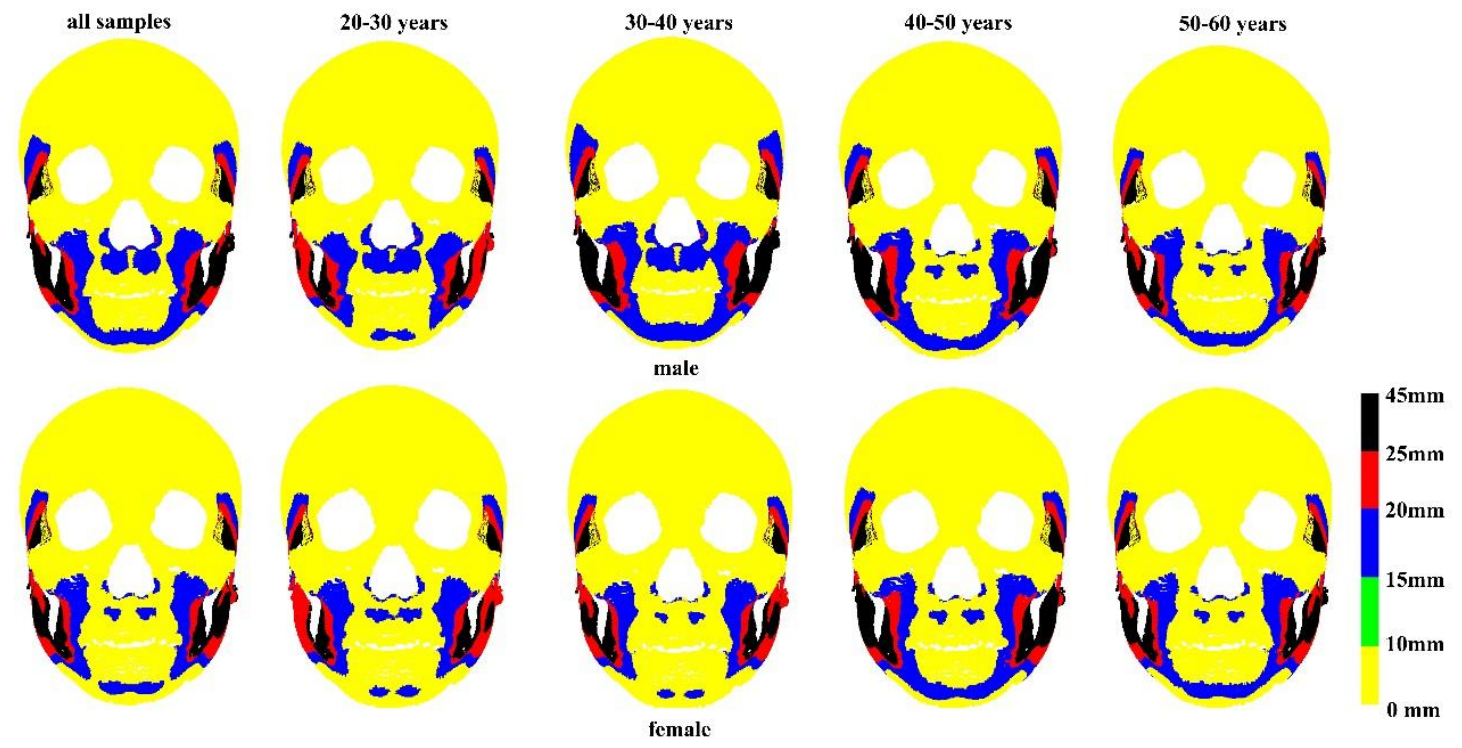

Fig. 8. Average densely calculated FSTT for each category according to sex and age.
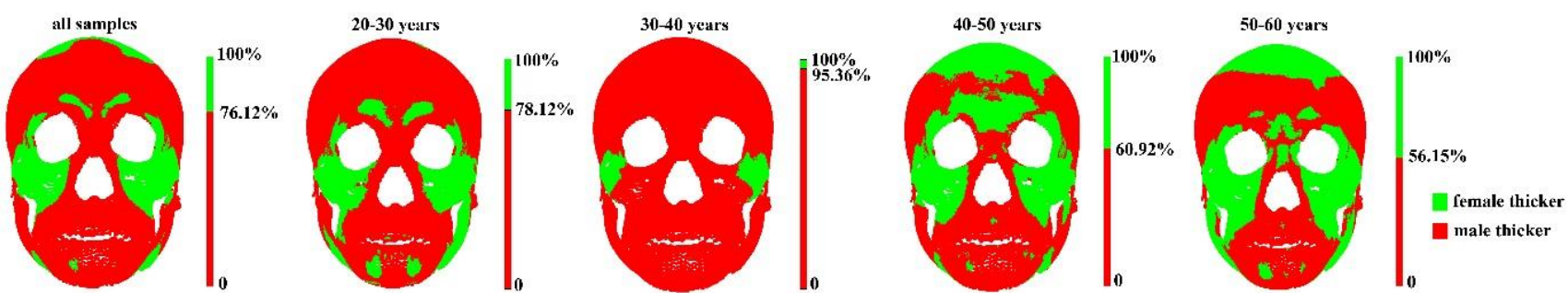

Fig. 9. Comparison between males and females in the same age groups. 


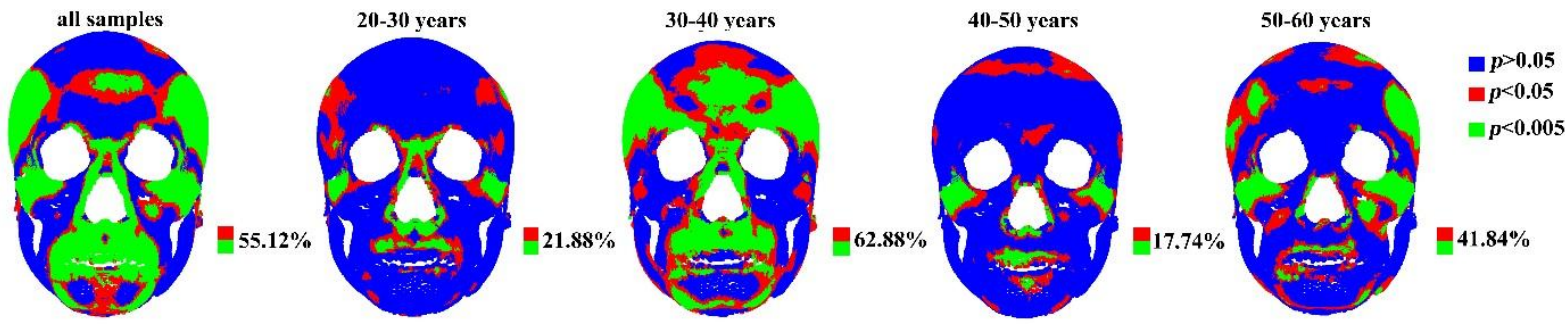

Fig. 10. Significant differences between males and females in the same age groups.
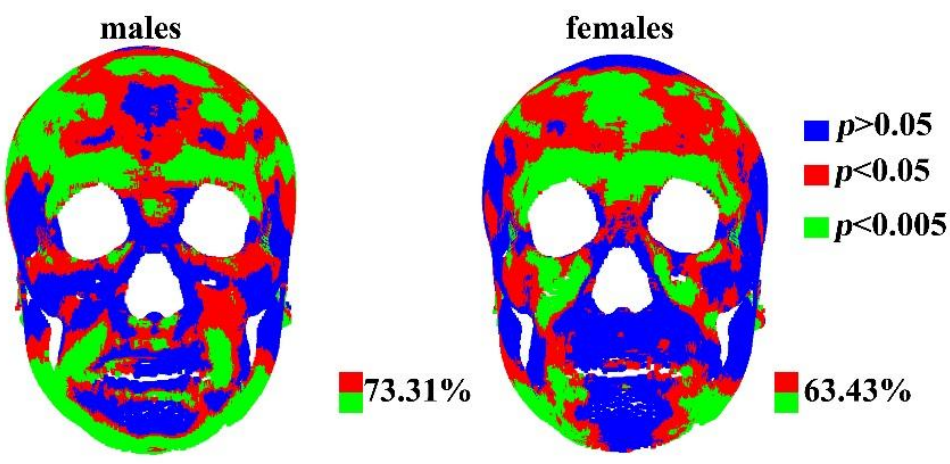

Fig. 11. Significant differences between the three age groups of males and females.
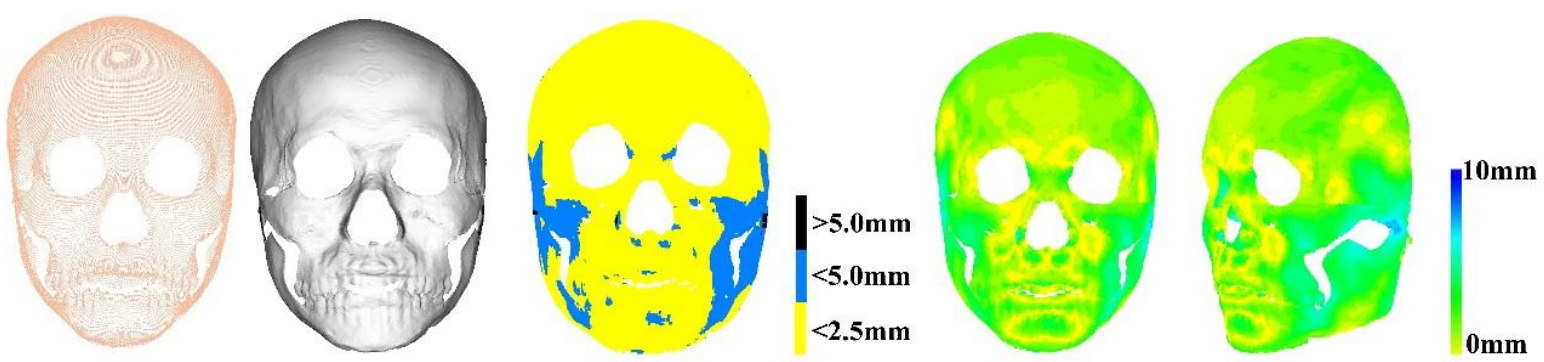

Fig. 12. The example of craniofacial reconstruction using densely calculated FSTT in the male 20-30 years group. 


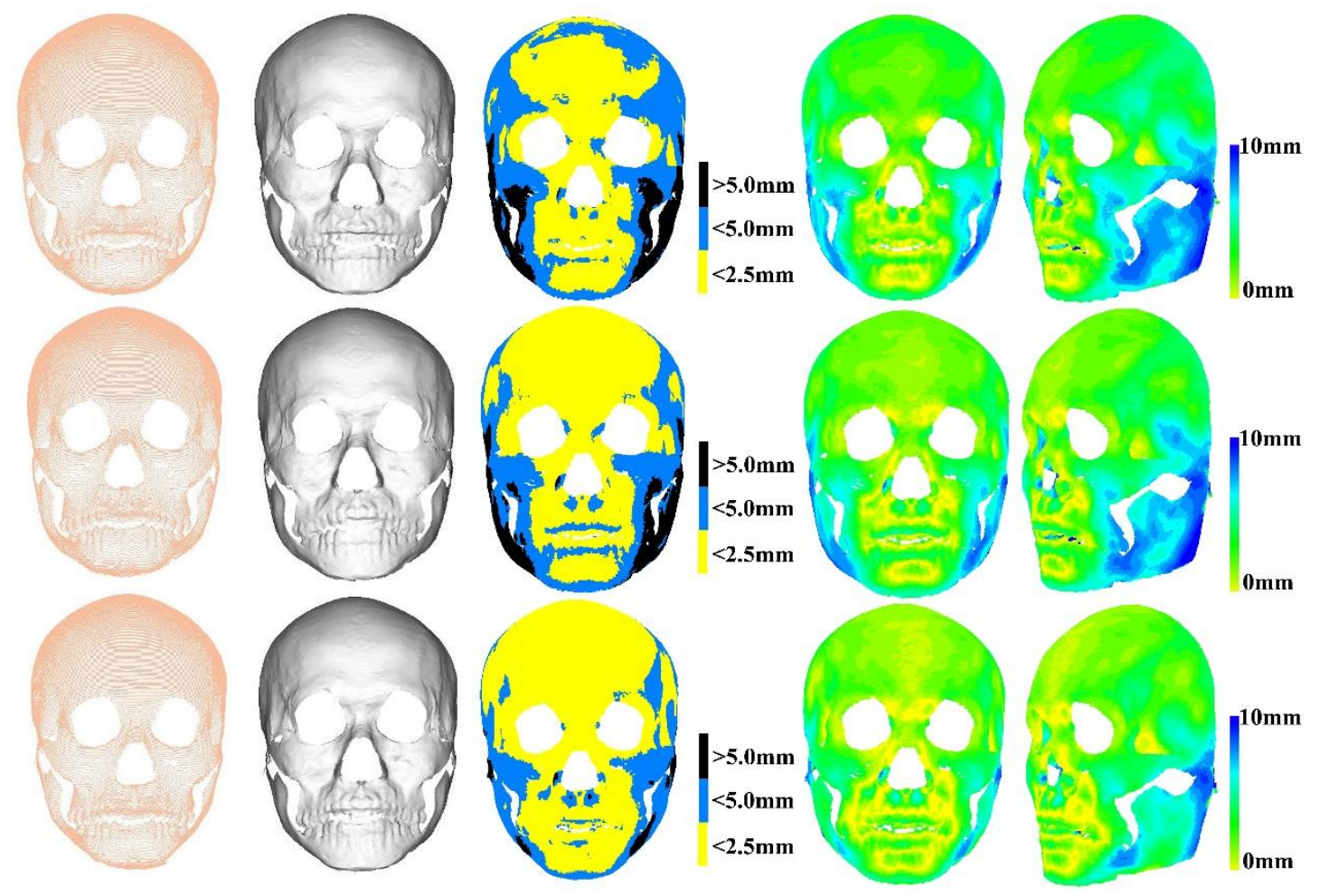

Fig. 13. Geometric deviation between reconstructed appearances using the average densely calculated FSTT of male groups and the actual appearance. From top to bottom, the figures are respectively ages 30-40 years, 40-50 years and 50-60 years. 


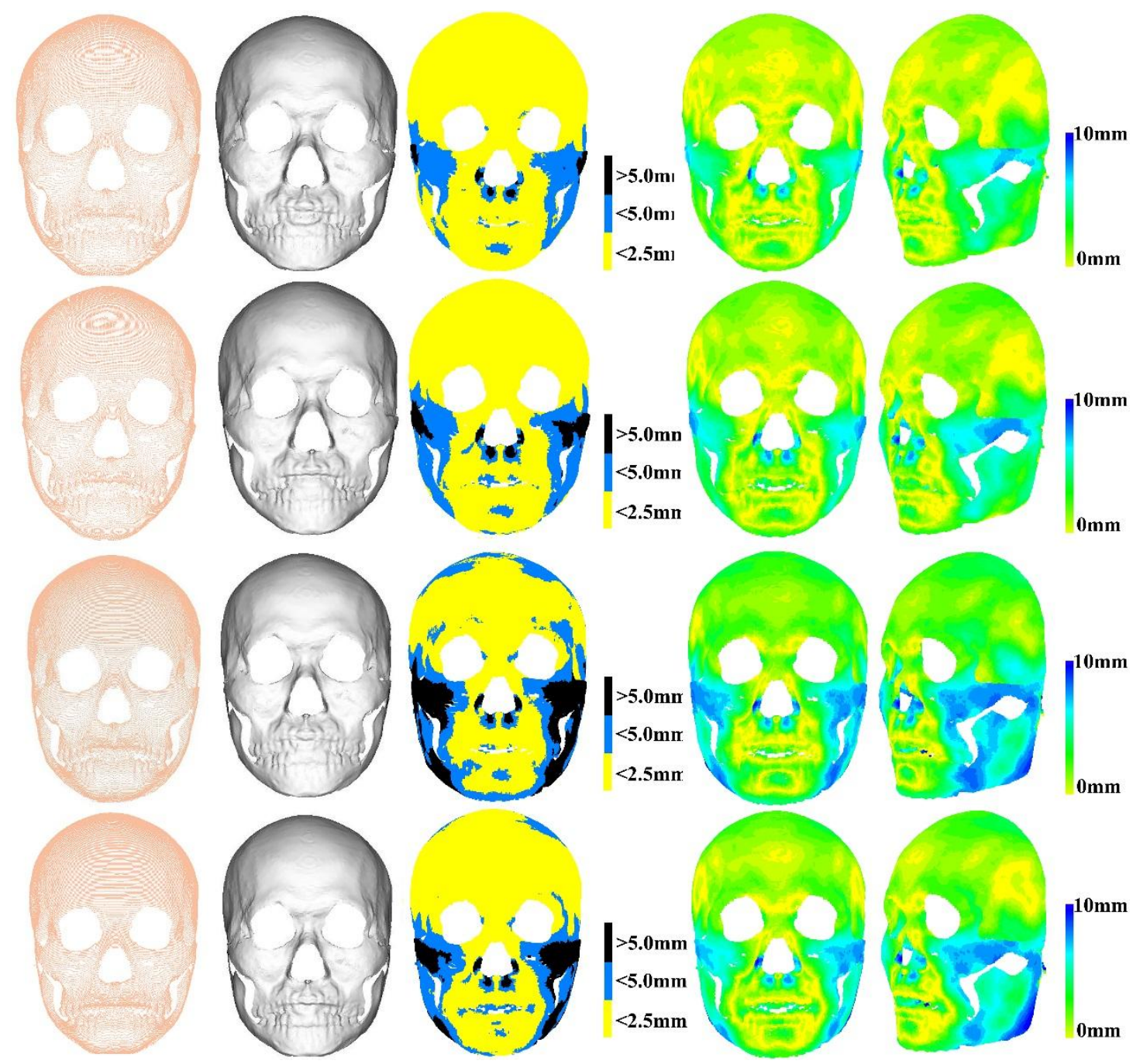

Fig. 14. Geometric deviation between reconstructed appearance using the average densely calculated FSTT of female groups and the actual appearance. From top to bottom, the figures are respectively 20-30 years, 30-40 years, 40-50 years and 50-60 years.
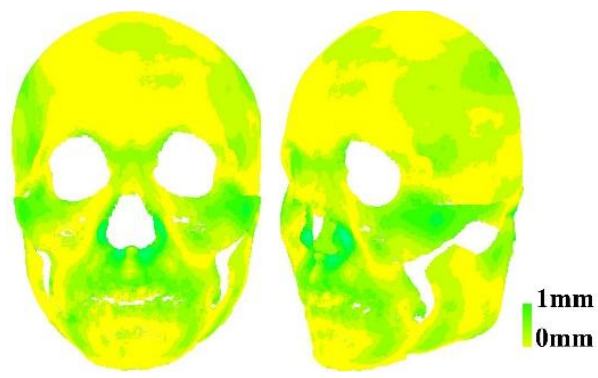

Fig. 15. Geometric deviation using average densely calculated FSTT of the 20-30 years group between male and female. 
Table 1 Comparison of descriptive statistics between Chinese males and females in each category.

\begin{tabular}{|c|c|c|c|c|c|c|c|c|}
\hline \multirow{2}{*}{ Category } & \multicolumn{4}{|c|}{ Male $(n=100)$} & \multicolumn{4}{|c|}{ Female $(n=71)$} \\
\hline & SMean & SSD & LSD & $\%$ & SMean & $S S D$ & LSD & $\%$ \\
\hline category 1 & 5.92 & 1.48 & 1.68 & $48.6 \%$ & 5.79 & 1.44 & 1.76 & $49.8 \%$ \\
\hline category 2 & 12.51 & 2.65 & 1.41 & $20.0 \%$ & 12.26 & 2.10 & 1.44 & $22.4 \%$ \\
\hline category 3 & 17.16 & 3.29 & 1.42 & $13.7 \%$ & 17.20 & 2.75 & 1.49 & $11.4 \%$ \\
\hline category 4 & 22.70 & 4.22 & 1.44 & $10.3 \%$ & 22.57 & 3.21 & 1.40 & $10.6 \%$ \\
\hline category 5 & 27.72 & 3.98 & 2.89 & $7.4 \%$ & 27.88 & 2.98 & 3.13 & $5.8 \%$ \\
\hline
\end{tabular}

Table 2 Comparison of descriptive statistics among age groups for males in each category.

\begin{tabular}{|c|c|c|c|c|c|c|c|c|c|c|c|c|c|c|c|c|}
\hline \multirow{2}{*}{ Category } & \multicolumn{4}{|c|}{$20-30$ years $(n=30)$} & \multicolumn{4}{|c|}{$30-40$ years $(n=25)$} & \multicolumn{4}{|c|}{$40-50$ years $(n=25)$} & \multicolumn{4}{|c|}{$50-60$ years $(n=20)$} \\
\hline & SMean & SSD & LSD & $\%$ & SMean & SSD & LSD & $\%$ & SMean & SSD & LSD & $\%$ & SMean & SSD & LSD & $\%$ \\
\hline category 1 & 5.62 & 1.24 & 1.83 & $53.4 \%$ & 6.34 & 1.46 & 1.49 & $44.6 \%$ & 5.95 & 1.26 & 1.65 & $46.7 \%$ & 5.77 & 1.17 & 1.76 & $49.4 \%$ \\
\hline category 2 & 12.33 & 2.20 & 1.46 & $20.2 \%$ & 12.52 & 2.30 & 1.43 & $20.4 \%$ & 12.56 & 2.31 & 1.40 & $18.7 \%$ & 12.52 & 2.24 & 1.39 & $20.2 \%$ \\
\hline category 3 & 17.38 & 2.70 & 1.49 & $13.0 \%$ & 17.15 & 2.98 & 1.38 & $15.2 \%$ & 17.22 & 2.90 & 1.38 & $14.3 \%$ & 17.12 & 2.82 & 1.38 & $13.6 \%$ \\
\hline category 4 & 22.50 & 2.91 & 1.47 & $10.1 \%$ & 22.51 & 3.65 & 1.50 & $9.0 \%$ & 22.59 & 3.75 & 1.51 & $8.4 \%$ & 22.74 & 3.41 & 1.44 & $10.3 \%$ \\
\hline category 5 & 28.37 & 3.00 & 2.85 & $3.37 \%$ & 27.92 & 3.79 & 2.90 & $10.9 \%$ & 27.93 & 4.07 & 2.86 & $11.9 \%$ & 27.62 & 3.69 & 2.76 & $6.6 \%$ \\
\hline
\end{tabular}

Table 3 Comparison of descriptive statistics among age groups for females in each category.

\begin{tabular}{|c|c|c|c|c|c|c|c|c|c|c|c|c|c|c|c|c|}
\hline \multirow{2}{*}{ Category } & \multicolumn{4}{|c|}{$20-30$ years $(n=15)$} & \multicolumn{4}{|c|}{$30-40$ years $(n=15)$} & \multicolumn{4}{|c|}{$40-50$ years $(n=16)$} & \multicolumn{4}{|c|}{$50-60$ years $(n=25)$} \\
\hline & SMean & SSD & LSD & $\%$ & SMean & SSD & LSD & $\%$ & SMean & SSD & LSD & $\%$ & SMean & SSD & LSD & $\%$ \\
\hline category 1 & 5.44 & 1.03 & 1.92 & $52.6 \%$ & 5.59 & 1.15 & 1.84 & $54.1 \%$ & 6.18 & 1.67 & 1.54 & $46.2 \%$ & 5.85 & 1.22 & 1.79 & $49.9 \%$ \\
\hline category 2 & 12.28 & 1.66 & 1.46 & $21.9 \%$ & 12.22 & 2.17 & 1.42 & $23.3 \%$ & 12.28 & 1.97 & 1.40 & $22.9 \%$ & 12.22 & 1.88 & 1.41 & $21.8 \%$ \\
\hline category 3 & 17.40 & 2.05 & 1.51 & $12.3 \%$ & 17.30 & 2.78 & 1.48 & $10.8 \%$ & 17.18 & 2.52 & 1.47 & $12.3 \%$ & 17.16 & 2.43 & 1.46 & $11.0 \%$ \\
\hline category 4 & 22.47 & 2.06 & 1.52 & $9.4 \%$ & 22.45 & 3.05 & 1.49 & $9.9 \%$ & 22.62 & 2.98 & 1.43 & $10.9 \%$ & 22.82 & 3.08 & 1.41 & $10.5 \%$ \\
\hline category 5 & 28.41 & 2.30 & 3.06 & $3.7 \%$ & 28.19 & 2.99 & 3.36 & $5.0 \%$ & 27.86 & 2.53 & 3.00 & $7.6 \%$ & 27.85 & 3.15 & 3.01 & $6.9 \%$ \\
\hline
\end{tabular}

Table 4 The percentage distribution (\%) and geometric deviation using average densely calculated FSTT of males in four age groups

\begin{tabular}{|c|c|c|c|c|c|c|c|c|}
\hline \multirow{2}{*}{ Error } & \multicolumn{2}{|c|}{ 20-30 years } & \multicolumn{2}{|c|}{$30-40$ years } & \multicolumn{2}{|c|}{$40-50$ years } & \multicolumn{2}{|c|}{$50-60$ years } \\
\hline & $\%$ & avgError(mm) & $\%$ & avgError(mm) & $\%$ & avgError(mm) & $\%$ & avgError(mm) \\
\hline$(0.0,2.5 \mathrm{~mm})$ & $79.3 \%$ & 1.37 & $34.6 \%$ & 1.78 & $49.7 \%$ & 1.62 & $60.7 \%$ & 1.44 \\
\hline$(2.5 \mathrm{~mm}, 5.0 \mathrm{~mm}]$ & $20.4 \%$ & 3.38 & $43.6 \%$ & 3.46 & $29.9 \%$ & 3.53 & $32.2 \%$ & 3.52 \\
\hline$>5.0 \mathrm{~mm}$ & $0.3 \%$ & 5.50 & $21.8 \%$ & 6.27 & $20.4 \%$ & 6.57 & $7.1 \%$ & 6.09 \\
\hline Total & $100 \%$ & 1.79 & $100 \%$ & 3.49 & $100 \%$ & 3.2 & $100 \%$ & 2.44 \\
\hline
\end{tabular}

Table 5 The percentage distribution (\%) and geometric deviation using average densely calculated FSTT of females in four age groups

\begin{tabular}{|c|c|c|c|c|c|c|c|c|}
\hline \multirow{2}{*}{ Error } & \multicolumn{2}{|c|}{ 20-30 years } & \multicolumn{2}{|c|}{$30-40$ years } & \multicolumn{2}{|c|}{$40-50$ years } & \multicolumn{2}{|c|}{$50-60$ years } \\
\hline & $\%$ & avgError(mm) & $\%$ & avgError(mm) & $\%$ & avgError(mm) & $\%$ & $\operatorname{avgError}(\mathrm{mm})$ \\
\hline$(0.0,2.5 \mathrm{~mm})$ & $80.0 \%$ & 1.05 & $73.7 \%$ & 1.23 & $50.4 \%$ & 1.56 & $63.8 \%$ & 1.31 \\
\hline$(2.5 \mathrm{~mm}, 5.0 \mathrm{~mm}]$ & $18.0 \%$ & 3.54 & $22.2 \%$ & 3.63 & $29.8 \%$ & 3.46 & $23.2 \%$ & 3.67 \\
\hline$>5.0 \mathrm{~mm}$ & $2.0 \%$ & 5.53 & $4.1 \%$ & 5.73 & $19.8 \%$ & 6.2 & $13.0 \%$ & 6.29 \\
\hline Total & $100 \%$ & 1.59 & $100 \%$ & 1.95 & $100 \%$ & 3.04 & $100 \%$ & 2.50 \\
\hline
\end{tabular}

